\title{
Rigorous 3D Electrostatic Field Analysis of SAW Transducers with Closed-Form Formulae
}

\author{
A.R. BAGHAI-WADJI, S. SELBERHERR AND F. SEIFERT
}

\author{
Technische Universität Wien \\ Institut für Allgemeine Elektrotechnik u. Elektronik \\ Gußhausstraße 27-29/A-1040 Vienna, Austria
}

\begin{abstract}
The extreme requirements of SAW filter design for gtringent specifications demand the knowledge of the exact nonuniform charge distribution along the fingers, especially for narrow-aperture, curved-finger and for apodized transducers. To solve this problem we present a natural extension of our previously published work, developing a self-contained, comprehensive method for three dimensional (3D) electrostatic field analysis of SAW transducers. The method is based on an analytically formulated Green's function and utilizes the advantages of the method of moments. No restrictions have to be imposed on the substrate anisotropy properties, the 2D topology of the fingers and the potentials applied to specific finger groups. Much effort has been devoted to the solution of the problem with closed-form formulae making the method applicable to SAW filter design with a reasonable amount of computing time. Computational results of SAW structures on $\mathrm{YZ}-\mathrm{LiNbO}$, rotated $\mathrm{LiNbO}_{3}$ and $Q$ uart $z$ are presented, fully taking into account the influence of bus-bars. Considerable attention has been paid to the development of general guidelines for the design of SAW transducers. The dependence of the finger capacitances on the aperture width and on the anisotropic properties of the substrate are tabulated for commonly used materials.
\end{abstract}

\section{Introduction}

In recent years design and technology of surface acoustic wave (SAW) interdigital transducers have achieved such a high standard, that for many cases of greatest practical interest, like apodized transducers, curved- finger transducers and transducers with small apertures, the two- dimensional field analysis of SAW filters (one-dimensinnal charge distribution) cannot fulfill the imposed severe requirements. Therefore, the calculation of 3-dimensional field distribution (2-dimensional charge distribution) has become indispensible, especially for the accurate determination of the SAW diffraction pattern due to small overlap and to include the influence of the bus-bars on the charge distribution (transducer capacitance). As to our knowlegde the only serious work on this problem is due to Wagers [1]. In his work, Wagers assumes transducer structures with twodimensional periodicity. This fact reduces the range of applicability of his method to unrealistic configurations. On the other hand the resulting formulae contain a great number of double sums, which must be calculated numerically. As he has pointed out this process is very time-consuming. To overcome these restrictions we have developed a noniterative semi- numerical method for 3D electrostatic field analysis of arbitrary thin metallic structures deposited on the surface of an anisotropic semi- infinite dielectric.
The aim of this paper is to present a method with a wide range of applicability, as we already shown for simpler cases, $\{2],[3],[4]$.

Using the two-dimensional Green's function in the wavenumber domain [5], the spectral domain representation and the point-matching technique, a formalism with closed-form formulae has been developed.

In what follows, firstly we will establish the basic relations and discuss the method of analysis. Then, applying this technique, we will present and discuss some of selected results we have obtained. For $\mathrm{YZ}-\mathrm{LiNbO}_{3}, 128^{0}$ rot. $\mathrm{LiNbO}_{3}$ and $Q u a r t z$ the influence of the aperture length and of the bus-bars as well as of the anisotropy of the substrate is illustrated by some graphs.

\section{Theory}

Consider a semi-infinite anisotropic dielectric characterized by $(\underline{\epsilon}) .(\underline{\epsilon})$ is a three by three symmetric positive definite matrix $[4],[6]$, i.e.

$$
\begin{gathered}
(\underline{\underline{\epsilon}})=\epsilon_{0} \cdot\left(\begin{array}{ccc}
\epsilon_{11} & \epsilon_{12} & \epsilon_{13} \\
\epsilon_{12} & \epsilon_{22} & \epsilon_{23} \\
\epsilon_{13} & \epsilon_{23} & \epsilon_{33}
\end{array}\right) \\
\epsilon_{11}>0, \epsilon_{22}>0, \epsilon_{33}>0 \\
\epsilon_{11} \cdot \epsilon_{22}-\epsilon_{12}{ }^{2}>0 \\
\epsilon_{11} \cdot \epsilon_{33}-\epsilon_{13}{ }^{2}>0 \\
\epsilon_{22} \cdot \epsilon_{33}-\epsilon_{23}{ }^{2}>0 \\
\operatorname{det}(\underline{\underline{\epsilon}})>0
\end{gathered}
$$

A set of infinitely thin electrodes (fingers) may be deposited on the plane surface of the substrate. There are no restrictions imposed on the 2D-geometry of the fingers and on the finger potentials. The fingers are assumed to have negligible sheet resistivity, Fig.1.

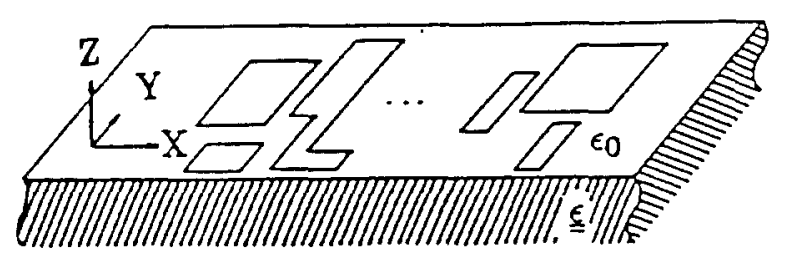

Fig.1 Geometry of interest 
With regard to the above conditions and assuming a time variation as $e^{j \omega t}$, we will now formulate the problem and establish the basic equations.

The problem is to find an efficient method for the calculation of the three-dimensional field distribution for configurations as sketched in Fig.1. As we will see, the whole field problem is uniquely determined if the charge distribution on the fingers is evaluated. This is due to the fact that the active zone, which controls the field distribution only resides on the interface.

In free space the Maxwell Eqs. (7), (9) and the constitutive Eq. (8) are valid

$$
\begin{gathered}
\vec{E}_{f}=-\vec{\nabla} \phi_{f} \\
\vec{D}_{f}=\epsilon_{0} \cdot \vec{E}_{f} \\
\vec{\nabla} \cdot \vec{D}_{f}=0
\end{gathered}
$$

Correspondingly in the substrate we have

$$
\begin{aligned}
& \vec{E}_{s}=-\vec{\nabla} \phi_{s} \\
& \vec{D}_{s}=\underline{\epsilon} \cdot \vec{E}_{s} \\
& \vec{\nabla} \cdot \vec{D}_{s}=0
\end{aligned}
$$

The solution of the system of Eqs. (7)-(12) has to be subjected to the following boundary conditions:

i)

$$
\begin{aligned}
& \lim _{z \rightarrow \infty} \phi_{f}(x, y, z) \rightarrow 0 \\
& \lim _{z \rightarrow-\infty} \phi_{s}(x, y, z) \rightarrow 0
\end{aligned}
$$

Eqs. (13) and (14) ensure that the potential in the direction perpendicular to the free space-substrate interface vanishes for $|z| \rightarrow \infty$.

ii) The potential on the interface is continuous and must be bounded, (15).

$$
\phi_{f}\left(x, y, z=0^{+}\right)=\phi_{s}\left(x, y, z=0^{-}\right)=\Phi(x, y)
$$

$\Phi(x, y)$ denotes the spatial variation on the surface of the substrate.

iii) The difference between the normal components of the dielectric displacement on the interface equals the spatial distribution of the charge density (16)

$$
D_{z, f}\left(x, y, z=0^{+}\right)-D_{z, 8}\left(x, y, z=0^{-}\right) \quad \rho(x, y)
$$

Insertion of (7) in (8) and (8) in (9) results in the Laplace equation in free space

$$
\phi_{x x}+\phi_{y y}+\phi_{z z}=0
$$

In a similar manner we obtain the Laplace equation for the substrate

$$
\begin{aligned}
\epsilon_{11} \phi_{x x} & +\epsilon_{22} \phi_{y y}+\epsilon_{33} \phi_{z z}+ \\
& +2 \epsilon_{12} \phi_{x y}+2 \epsilon_{13} \phi_{x z}+2 \epsilon_{23} \phi_{y z}=0
\end{aligned}
$$

By substitution one can show that solutions of the form

$$
\phi_{f}(x, y, z)=A\left(k_{x}, k_{y}\right) e^{-j k_{x} x} e^{-j k y} e^{\gamma z z}
$$

with

$$
\gamma_{f}\left(k_{x}, k_{y}\right)=-\sqrt{k_{x}^{2}+k_{y}^{2}}
$$

satisfy (17). Because of the linearity of the system of equations, the superposition principle holds and we get as a general solution for the Laplace equation in the free space

$$
\begin{aligned}
\phi_{f}(x, y, z)= & \left(\begin{array}{c}
1 \\
2 \pi
\end{array}\right)^{2} \int_{-\infty}^{+\infty} \int_{-\infty}^{+\infty} \bar{A}\left(k_{x}, k_{y}\right) . \\
& e^{-j k_{x} x} e^{-j k_{y} y} e^{\gamma f^{z}} d k_{x} d k_{y}
\end{aligned}
$$

Similarly one can show that solutions of the form

$$
\phi_{s}(x, y, z)=\bar{B}\left(k_{x}, k_{y}\right) e^{-j k_{x} x} e^{-j k_{y} y} e^{\gamma_{x} z}
$$

with

$$
\begin{aligned}
\gamma_{s}\left(k_{x}, k_{y}\right) & =j \frac{\epsilon_{13} 3}{\epsilon_{33}} k_{x}+j \frac{\epsilon_{23}}{\epsilon_{33}} k_{y}+ \\
& +\sqrt{\epsilon_{r 1}^{2}} k_{x}^{2}+2 \epsilon_{r 12} k_{x} k_{y}+\epsilon_{r 2}^{2} k_{y}^{2}
\end{aligned}
$$

and

$$
\begin{aligned}
\epsilon_{r 1}^{2} & =\frac{\epsilon_{11} \epsilon_{33}-\epsilon_{13}^{2}}{\epsilon_{33}^{2}} \\
\epsilon_{r 2}^{2} & =\frac{\epsilon_{22} \epsilon_{33}-\epsilon_{23}^{2}}{\epsilon_{33}^{2}} \\
\epsilon_{r 12} & =\frac{\epsilon_{12} \epsilon_{33}-\epsilon_{13} \epsilon_{33}}{\epsilon_{33}^{2}}
\end{aligned}
$$

satisfy the Laplace equation in the substrate, which leads to the general solution.

$$
\phi_{s}(x, y, z)=\left(\begin{array}{c}
1 \\
2 \pi
\end{array}\right)^{2} \int_{-\infty}^{+\infty} \int_{-\infty}^{+\infty} \bar{B}\left(k_{x}, k_{z}\right) \cdot e^{-j k_{x} z} e^{-j k_{y} y} e^{\gamma_{f} z} d k_{x} d k_{y}
$$

Now let us identify the physical meaning of $\bar{A}\left(k_{z}, k_{y}\right)$ and $B\left(k_{x}, k_{y}\right)$.

For this purpose we specify $\phi_{f}(x, y, z)$ and $\phi_{s}(x, y, z)$, respectively, for the limit cases $z \rightarrow 0^{+}$and $z \rightarrow 0^{-}$, obtainig (28) and (29)

$$
\begin{aligned}
\phi_{f}\left(x, y, z=0^{+}\right)= & \left(\frac{1}{2 \pi}\right)^{2} \int_{-\infty}^{+\infty} \int_{-\infty}^{+\infty} A\left(k_{r}, k_{y}\right) . \\
& e^{-j k_{x} x} e^{-j k_{y} y} d k_{x} d k_{y} \\
\phi_{s}\left(x, y, z=0^{-}\right)= & \left(\frac{1}{2 \pi}\right)^{2} \int_{-\infty}^{+\infty} \int_{-\infty}^{+\infty} \bar{B}\left(k_{x}, k_{y}\right) . \\
& \cdot e^{-j k_{x} x} e^{-j k_{y} y} d k_{x} d k_{y}
\end{aligned}
$$

In accordance with (15) $\phi_{f}\left(x, y, z=0^{+}\right)$and $\phi_{s}(x, y, z=$ $0^{-}$) at the left hand side of (28) and (29) are equal to $\Phi(x, y)$, which can be written in the form

$$
\Phi(x, y)=\left(\frac{1}{2 \pi}\right)^{2} \int_{-\infty}^{+\infty} \int_{-\infty}^{+\infty} \bar{\phi}\left(k_{x}, k_{y}\right) e^{-j k_{x} x} e^{-j k_{y} y} d k_{x} d k_{y}
$$

Consequently, (28) and (29) represent the two-dimensional inverse Fourier transformation of the potential distribution on the interface and we obtain

$$
\bar{A}\left(k_{x}, k_{y}\right)=\bar{B}\left(k_{x}, k_{y}\right)=\phi\left(k_{x}, k_{y}\right)
$$

That means $\bar{A}\left(k_{x}, k_{y}\right)$ and $\bar{B}\left(k_{x}, k_{y}\right)$ are equal to the spectral components of the potential distribution on the surface. Using (7), (8), (21) and (10), (11), (27) together with (31) and the boundary condition (16) we have 


$$
\bar{\phi}\left(k_{x}, k_{y}\right)=\bar{G}\left(k_{x}, k_{y}\right) \cdot \bar{\rho}\left(k_{x}, k_{y}\right)
$$

with

$$
\bar{G}\left(k_{x}, k_{y}\right)=\frac{1}{\epsilon_{0}} \cdot \frac{1}{\sqrt{k_{x}^{2}}+k_{y}^{2}+\sqrt{\epsilon_{r 1}^{2} k_{x}^{2}+2 \epsilon_{r 12} k_{x} k_{y}+\epsilon_{r 2}^{2} k_{y}^{2}}}
$$

For $\rho(x, y)$ in $(16)$ we have used

$$
\rho(x, y)=\left(\frac{1}{2 \pi}\right)^{2} \int_{-\infty}^{+\infty} \int_{-\infty}^{+\infty} \bar{\rho}\left(k_{x}, k_{y}\right) e^{-j k_{x} x} e^{-j k_{y} y} d k_{x} d k_{y}
$$

$\bar{G}\left(k_{x}, k_{y}\right)$ is the Fourier transform of the potential distribution on the interface if a point charge source located on the interface excites the medium, Fig.2.

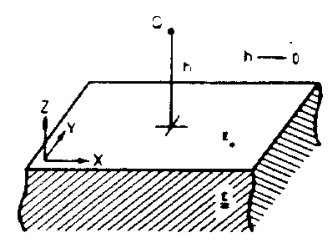

Fig. 2 Point charge source $(\delta-)$ excitation of a semi-infinite anisotropic dielectric

The response of a linear system to a $\delta$-disturbance is called the Green's function [7]. Therefore $\bar{G}\left(k_{x}, k_{i t}\right)$ is the Green's function in $\vec{k}$-domain, fully characterizing the boundary value problem sketched in Fig.1. In [4] we have extensively discussed the properties of $\bar{G}\left(k_{x}, k_{y}\right)$. It can also be shown that $\bar{G}\left(k_{x}, k_{y}\right)$ directly can be Fourier transformed into $G(x, y)$, if one interprets the associated inverse Fourier integral in a distributional sense. Note that by means of Eq.(32) we have found an expression for the spectral components of the potential on the surface, $\bar{\phi}\left(k_{x}, k_{y}\right)$. As is seen from (32), $\bar{\phi}\left(k_{x}, k_{y}\right)$ is composed of two parts, $\bar{G}\left(k_{x}, k_{y}\right)$ and $\bar{\rho}\left(k_{x}, k_{y}\right)$. While the first part explicitly is known (33), we have to find an approximate formula for $\bar{\rho}\left(k_{x}, k_{y}\right)$, which is simply realized in section IV. At the present stage of discussion it is sufficient to assume, that such an approximation exists

$$
\bar{\rho}\left(k_{x}, k_{y}\right)=\sum_{l=1}^{N} \sigma_{l} \bar{\rho}_{l}\left(k_{x}, k_{y}\right)
$$

where $\sigma_{l}$ are the unknown charge values (see section IV). Note that up to this point we have used two separate expressions for the potential in free space and within the substrate, respectively, (21) and (27). To have an unified formula for the potential in the whole $(x, y, z)$-space it is neccessary to introduce the following function

$$
\psi\left(z, k_{x}, k_{v}\right)=\gamma_{f}\left(k_{x}, k_{y}\right) \cdot H(z)+\gamma_{s}\left(k_{x}, k_{y}\right) \cdot H(-z)
$$

where $H(z)$ is the Heaviside step function. Using (21), (27) and (36) we obtain (37), which is valid in free space and within the substrate

$$
\begin{aligned}
\phi(x, y, z)= & \left(\frac{1}{2 \pi}\right)^{2} \int_{-\infty}^{+\infty} \int_{-\infty}^{+\infty} \bar{\phi}\left(k_{x}, k_{y}\right) . \\
& \cdot e^{-j k_{x} x} e^{-j k_{y} y} e^{\psi\left(z, k_{x}, k_{y}\right)} d k_{x}, d k_{y}
\end{aligned}
$$

(32) together with (35) yields

$$
\bar{\phi}\left(k_{x}, k_{y}\right)=\sum_{l=1}^{N} \sigma_{l} \bar{G}\left(k_{x}, k_{y}\right) p_{l}\left(k_{x}, k_{y}\right)
$$

which is an approximation for $\bar{\phi}\left(k_{x}, k_{y}\right)$. Insertion of (38) in (37) and interchanging the order of summation and integration yield

$$
\begin{aligned}
\phi(x, y, z)= & \sum_{l=1}^{N} \sigma_{l} \cdot\left(\begin{array}{c}
1 \\
2 \pi
\end{array}\right)^{2} \int_{-\infty}^{+\infty} \int_{-\infty}^{+\infty} \bar{G}\left(k_{x}, k_{y}\right) \bar{\rho}_{l}\left(k_{x}, k_{y}\right) . \\
& \cdot e^{-j k_{x} x} e^{-j k_{y} y} e^{\psi\left(z, k_{x}, k_{y}\right) z} d k_{x} d k_{y}
\end{aligned}
$$

Eq. (39) is the most important equation in our formalism. Once $\sigma_{l}$ has been evaluated, the whole field problem is determined uniquely. This is easily seen from (39) as follows: Firstly, by means of (39) the potential $\phi(x, y, z)$, and then, applying (7), (8), (10) and (11), the electrical field $\vec{E}(x, y, z)$ and the electrical displacement $\vec{D}(x, y, z)$ in the whole $(x, y, z)$-space can be calculated in a simple postproccessing step.

\section{Solution procedure}

Due to the fact that the potential values only on metalized regions on the interface $(x, y, z=0)$ are given, the next logical step is to have an expression for the potential distribution

on the interface, which is valid for every $(x, y)$ - point on the interface. Using (39) and setting $z=0$ we obtain

$$
\begin{aligned}
\Phi(x, y)= & \sum_{l=1}^{N} \sigma_{l}\left(\frac{1}{2 \pi}\right)^{2} \int_{-\infty}^{+\infty} \int_{-\infty}^{+\infty} G\left(k_{x}, k_{y}\right) \bar{\rho}_{l}\left(k_{x}, k_{y}\right) . \\
& e^{-j k_{x} x} e^{-j k_{y} y} d k_{x} d k_{y}
\end{aligned}
$$

In (40) we now need an explicit expression for $\bar{\rho}_{l}\left(k_{x}, k_{y}\right)$. The next section is devoted to this problem.

\section{Approximation of the charge density}

In connection with the collocation method (point-matching technique), which in the next section will be applied to (40), the commonly used basic functions for the approximation of the unknown function (here charge density) are the pulse function, step function and triangle approximations. In this paper we will use the stepfunction approximation. In order to take into account the finger-edge singularities of the charge distribution and in order to reduce the computer resources we have used a non-equidistance discretization of the fingers into subsquares, which is appropriate to our problem. Assuming that the transducer consists of $\mathrm{N}$ subsquares, and using the stepfunction approximation we obtain the following expression for the charge density

$$
\rho(x, y)=\rho_{0} \sum_{l=1}^{N} \sigma_{i} f_{l}(x, y)
$$

wherein $\rho_{0}$ is a normalization faktor, $\sigma_{l}$ is the unknown value of the charge density on the $l^{t h}$ subsquare and $f_{l}(x, y)$ is defined as follows 


$$
\begin{aligned}
f_{l}(x, y)= & {\left[H\left(x-x_{l}^{b}\right\}-H\left(x-x_{l}^{e}\right)\right] . } \\
\cdot & {\left[H\left(y-y_{l}^{b}\right)-H\left(y-y_{l}^{e}\right)\right] }
\end{aligned}
$$

$x_{l}^{b}, x_{l}^{e}$ and $y_{l}^{b}, y_{l}^{e}$ are the begin and end coordinates of the $l^{t h}$ subsquare. Inserting (41) in (43)

$$
\bar{\rho}\left(k_{x}, k_{y}\right)=\int_{-\infty}^{+\infty} \int_{-\infty}^{+\infty} \rho(x, y) e^{j k_{x} x} e^{j k_{y} y} d x d y
$$

we obtain

$$
\bar{\rho}\left(k_{x}, k_{y}\right)=\sum_{l=1}^{N} \sigma_{l} \vec{\rho}_{l}\left(k_{x}, k_{y}\right)
$$

with

$$
\bar{\rho}_{l}\left(k_{x}, k_{y}\right)=\rho_{0} \frac{-e^{-j a}+e^{-j b}+e^{-j c}-e^{-j d}}{k_{x} k_{y}}
$$

where

$$
\begin{aligned}
& a=k_{x} \cdot\left(\xi_{l}^{m}+\Delta \xi_{l}\right)+k_{y} \cdot\left(\eta_{l}^{m}+\Delta \eta_{l}\right) \\
& b=k_{x} \cdot\left(\xi_{l}^{m}+\Delta \xi_{l}\right)+k_{y} \cdot\left(\eta_{l}^{m}-\Delta \eta_{l}\right) \\
& c=k_{x} \cdot\left(\xi_{l}^{m}-\Delta \xi_{l}\right)+k_{y} \cdot\left(\eta_{l}^{m}+\Delta \eta_{l}\right) \\
& d=k_{x} \cdot\left(\xi_{l}^{m}-\Delta \xi_{l}\right)+k_{y} \cdot\left(\eta_{l}^{m}-\Delta \eta_{l}\right)
\end{aligned}
$$

$\xi_{l}^{m}$ and $\eta_{l}^{m}$ are the mid-point coordinates of the $l^{\text {th }}$ subsquare. $2 \cdot \Delta \xi_{l}$ and $2 \cdot \Delta \eta_{l}$ denote the $\mathrm{x}$ - and $\mathrm{y}$-extensions of the $l^{\text {th }}$ subsquare.

(44) is the aforementioned approximate formula for the $\bar{\rho}\left(k_{x}, k_{y}\right)$.

\section{V.Approximation of the potential distri- bution on the interface in closed-form}

Insertion of (44) in (39) leads to integrals which can be evaluated in closed-form. As a result we obtain

$$
\Phi(x, y)=\frac{\rho_{0}}{2 \pi \epsilon_{0}} \sum_{l=1}^{N} \sigma_{l} \cdot \ddot{A}_{l}(x, y)
$$

From a computational point of view it is more efficient to regard the charge integrals $\sigma_{l} \Delta \xi_{l} \Delta \eta_{l}$ as the unknowns rather than the charge values $\sigma_{l}$ themselves, which results in a better condition of the resulting matrix. That is due to the fact that we have used a non-equidistance discretization of the fingers. The higher the charge value on a subsquare, the smaller the area of the latter must be chosen to achieve values, of approximately the same order for the charge integrals. Taking this into account, $(50)$ can be the following form

$$
\Phi(x, y)=\sum_{l=1}^{N} \sigma_{l} \Delta \xi_{l} \Delta \eta_{l} \cdot \ddot{A}_{l}(x, y)
$$

with

$$
\begin{aligned}
A_{l}(x, y)=\underset{\Delta \xi_{l} \Delta \eta_{l}}{1} \cdot( & A_{l}^{(1)}(x, y)+A_{l}^{(2)}(x, y)+ \\
& \left.A_{l}^{(3)}(x, y)+A_{l}^{(4)}(x, y)\right)
\end{aligned}
$$

and

$$
\begin{aligned}
& A_{l}^{(1)}(x, y)=|a| \cdot I_{T}\left(\alpha, \beta, \gamma,-\frac{c}{a},-\frac{e}{a}\right) \\
& A_{l}^{(2)}(x, y)=|b| \cdot I_{T}\left(\alpha, \beta, \gamma,-\frac{e}{b},-\frac{c}{b}\right) \\
& A_{l}^{(3)}(x, y)=|c| \cdot I_{T}\left(\beta, \alpha, \gamma,-\frac{a}{c},-\frac{b}{c}\right) \\
& A_{l}^{(4)}(x, y)=|d| \cdot I_{T}\left(\beta, \alpha, \gamma,-\frac{b}{d},-\frac{a}{d}\right) \\
& a=x_{l}^{b}-x \\
& b=x_{l}^{e}-x \\
& c=y_{l}^{b}-y \\
& \quad d=y_{l}^{e}-y \\
& I_{T}\left(p_{1}, p_{2}, p_{3}, u_{1}, u_{2}\right)=\int_{u_{1}}^{u_{2}} \frac{1}{\sqrt{p_{1} u^{2}+2 p_{2} u+p_{3}}+\sqrt{u^{2}+1}} d u
\end{aligned}
$$

where we have used the scaling equation

$$
\frac{\rho_{0}}{2 \pi \epsilon_{0}}=1
$$

As we have discussed in [5] integrals of the type $I_{T}\left(p_{1}, p_{2}, p_{3}, u_{1}, u_{2}\right)$ can be solved in closed-form. Details about the results, and much more theoretical discussion of the method will be published soon.

\section{VI.Point-matching technique}

Because the potential values on the discretized subsquares are given

$$
\Phi\left(\xi_{k}, \eta_{k}\right)=\phi_{k}
$$

using the point-matching technique we obtain

$$
\Phi\left(\xi_{k}, \eta_{k}\right)=\sum_{l=1}^{N} \sigma_{l} \Delta \xi_{l} \Delta \eta_{l} \cdot{ }^{* *} \cdot A_{l}\left(\xi_{k}, \eta_{k}\right)
$$

or the same in matrix form

$$
\underline{\phi}=\stackrel{* *}{A} \cdot \underline{q}
$$

Inclusion of the charge neutrality condition in $[2]$ yields

$$
\underline{\phi}=\underline{\underline{A}} \cdot \underline{q}
$$

Finally, including the boundary conditions of the floating fingers, if such exist [2], we obtain

$$
\underline{\phi}=\underline{A} \cdot \underline{q}
$$

The matrix equation (63) corresponds to the CPIM, we have derived in $[2]$ for the one dimensional charge distribution

\section{Results}

Applying the above formalism, some of selected results will now be discussed. The first example (Fig.3) shows the influence of the discretization ( $\mathrm{M}$ is the number of subsquares on each finger) on the capacitance of the transducer considered. 


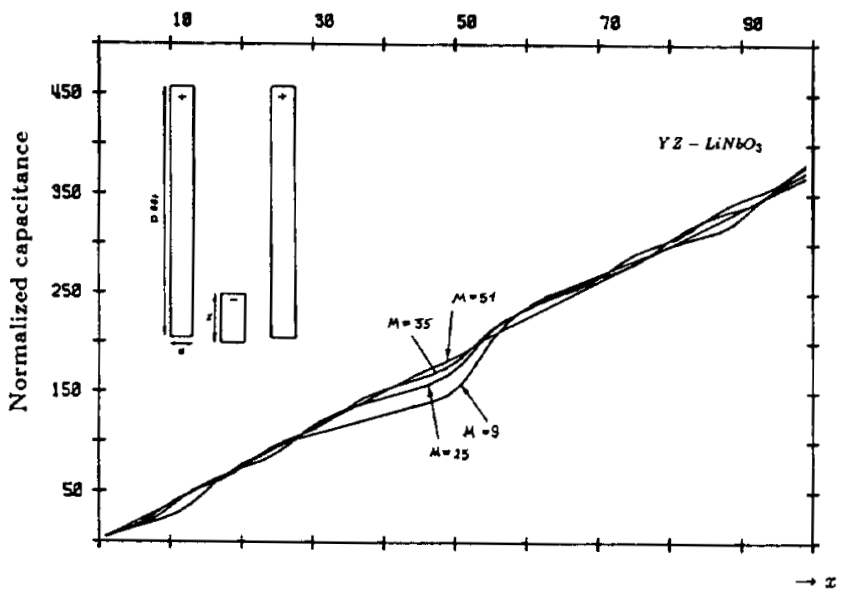

Fig.3 The influence of the discretization on the tranducer capacitance

Figures 4 and 5 show the normalized transducer capacitance as a function of the overlap-length for $\mathrm{YZ}-\mathrm{LiNbO}_{3}$, $128^{\circ}$ rot $-\mathrm{LiNbO}_{3}$ and Quartz.

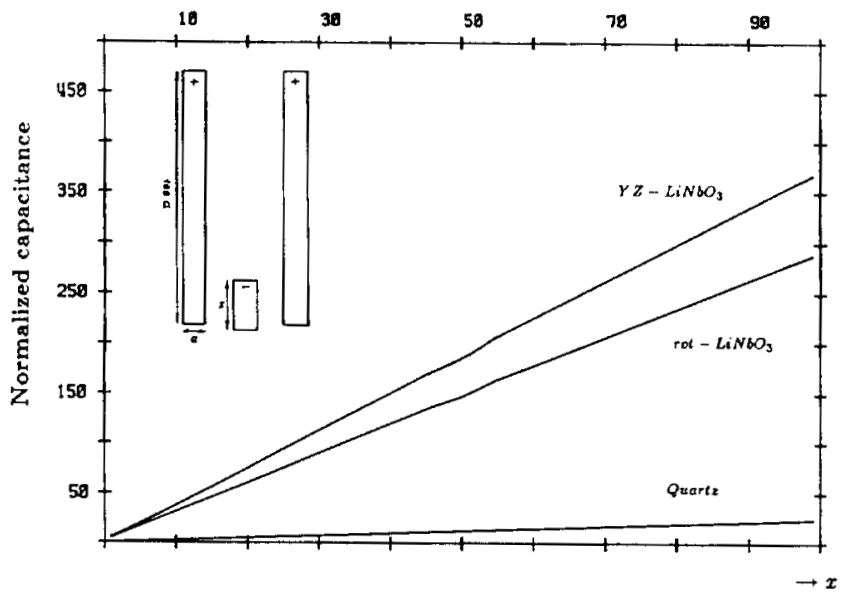

Fig.4 Normalized capacitance as a function of overlap-length

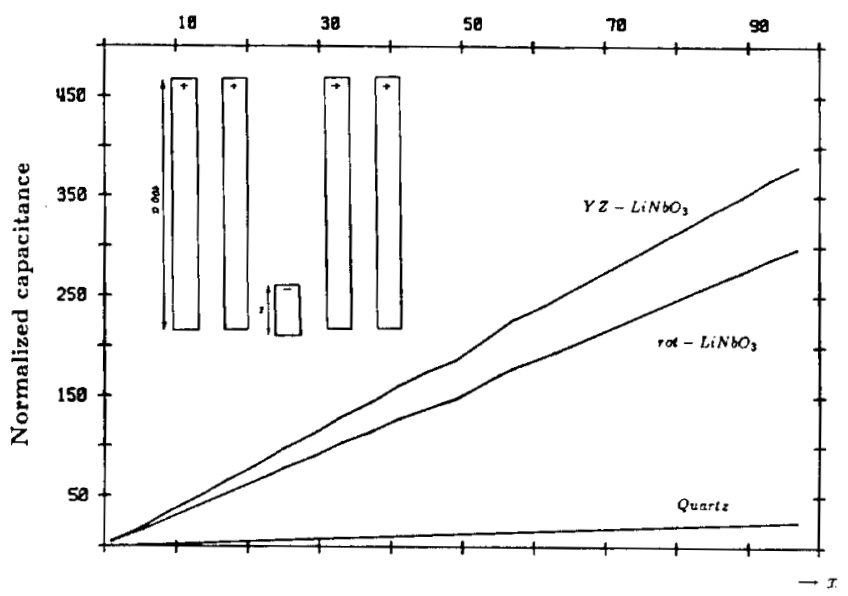

Fig.5 Normalized capacitance as a function of overlap-length
In Fig. 6 we see the influence of the bus-bars on the transducer capacitance.
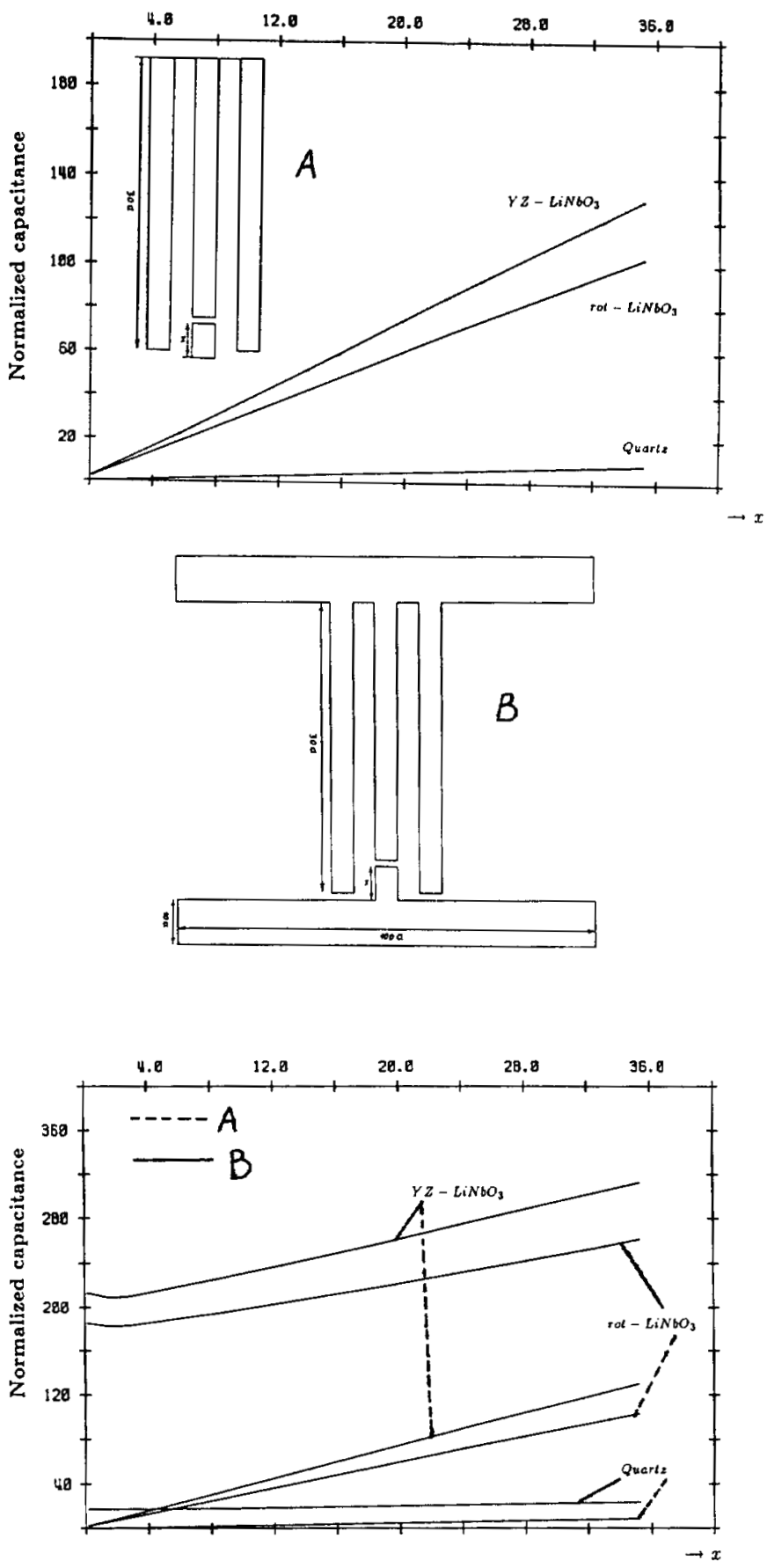

Fig. 6

Finally the next two examples may graphically demonstrate the influence of the bus-bars, as well as the transverse end effects. Both transducers are deposited on $\mathrm{YZ}-\mathrm{LiNbO}_{3}$. 

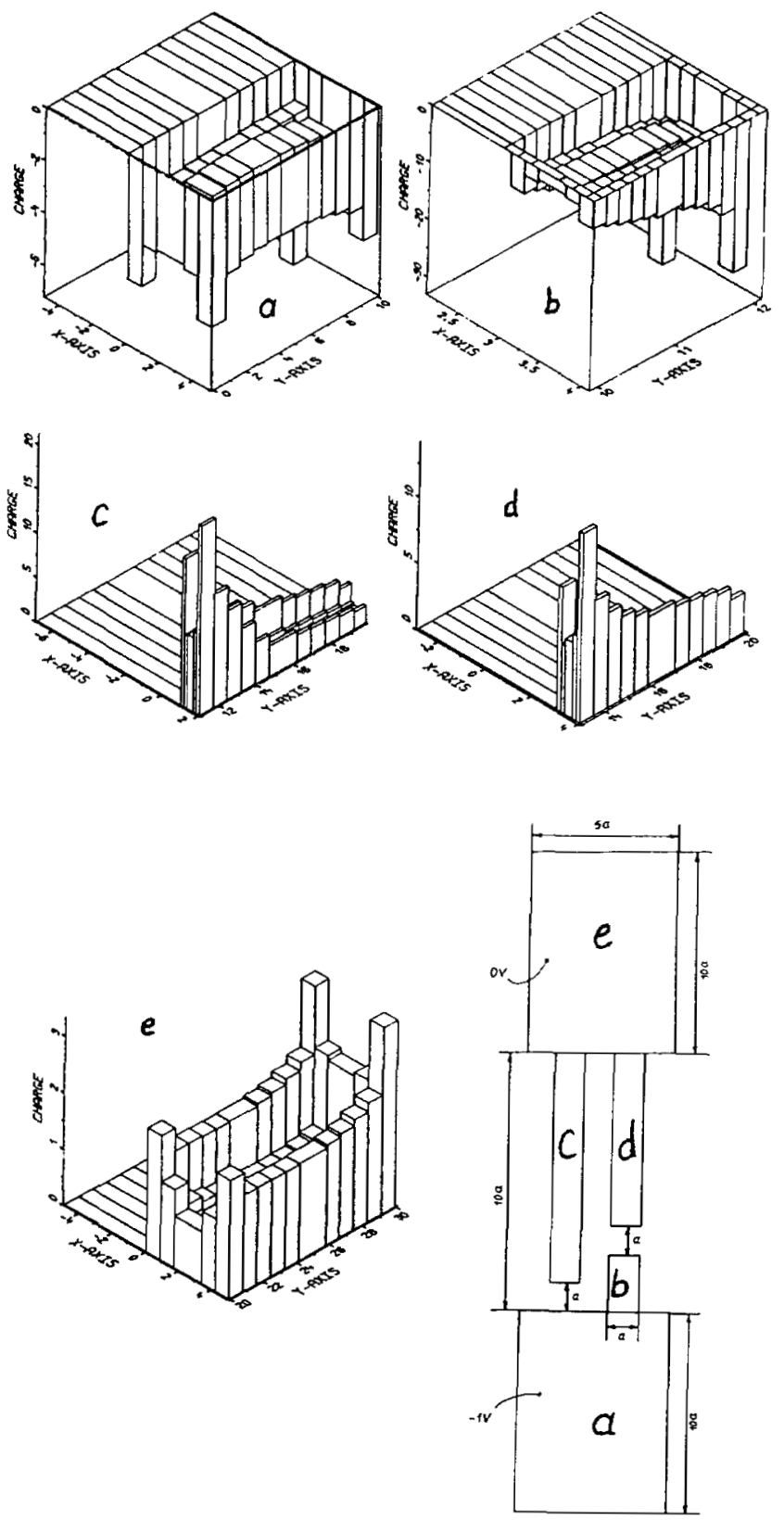

Fig.7 Charge distribution for a simple SAW structure on $\mathrm{YZ}-\mathrm{LiNbO}_{3}$

\section{Conclusion}

Using the Green's function concept, point-matching technique and spectral domain representation a self-contained, comprehensive method for three dimensional electrostatic field analysis of SAW transducers have been developed. The method is applied to SAW structures deposited on $\mathrm{YZ}-\mathrm{LiNbO}_{3}, 128$ rot $\mathrm{LiNbO}_{3}$ and Quartz. The influence of the bus-bars as well as the anisotropy of the substrate on the charge distribution (capacitance) are presented.
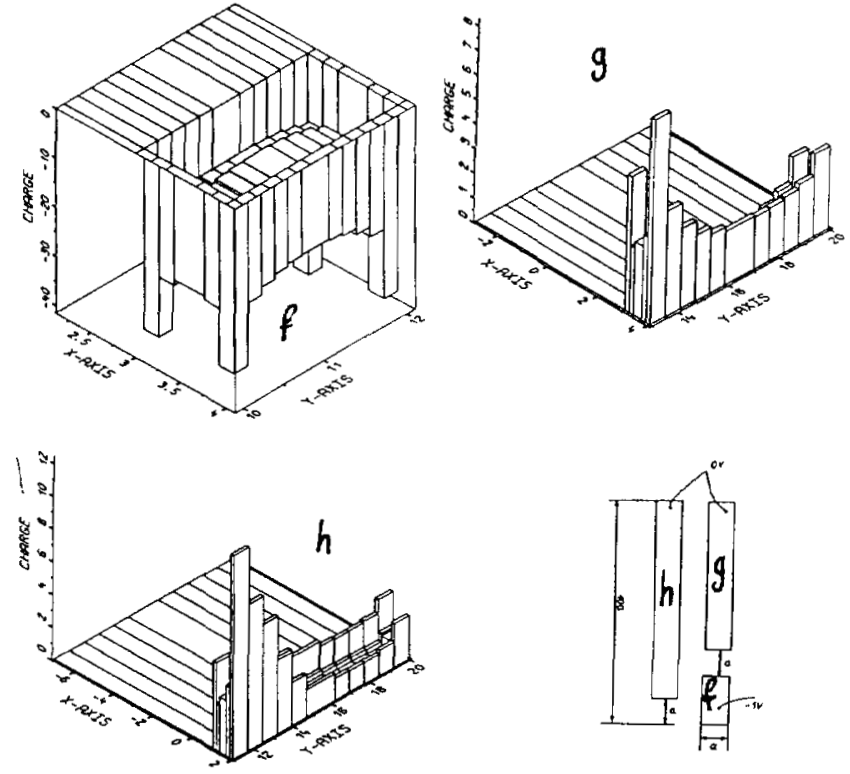

Fig.8 Charge distribution for The same transducer as in Fig.7, bus-bars included

\section{Acknowledgements}

This work is supported by Siemens AG, Central Research Laboratories, Munich, West Germany. Partial support by the Austrian Science Research Fund Project 5311 is acknowledged. Helpful discussions with Doz. M. Kowatsch are greatfully appriciated. Continuous encouragement and support by Dr. H Stocker are acknowledged.

\section{References}

(1) R.S. Wagers, Transverse electrostatic end effects in interdigital transducers, in Proc. Ultrason. Symp., 1976, pp.536-539

[2] A.R. Baghai-Wadji, S. Selberherr and F. Seifert, On the calculation of charge, electrostatic poptential and capacitance in generalized finite SAW structures, Proc. IEEE Ultrason. Symp., 1984, pp.44-48.

[3] ibid, Closed-form formulae analysis of SAW interaction with arbitrary interdigital transducer structures, in Proc. ISSWAS, Novosibirk, 1986

[4] ibid, A Green's function approach to the electrostatic problem of single, coupled and comb-like metallic structures in an isotropic multilayered media, Proc. AMSE, Sorrento, 1986

[5] ibid, Two-dimensional Green's function of a semiinfinite anisotropic dielectric in the wavenumber domain, IEEE Trans. on Ultrasonics, Ferroelectrics, and Frequency Controll, vol. UFFC-33, No. 3. May 1986

[6] M.H. Protter and H.F. Weinberger, Maximum principles in differential equations. New Jersey: Prentice-Hall, 1967, pp.56- 61.

(7) R.P. Kanwal, Generalized functions: Theory and technique, Academic Press, 1983, pp.52-56 